\title{
Diagnostic Efficacy of Endoscopic Ultrasound Elastography in Differentiating Solid Pancreatic Lesions: A Single-Center Experience
}

\author{
Ahmed Youssef Altonbary ${ }^{1}$, Hazem Hakim ${ }^{1}$ and Ahmed Mohamed El-Shamy ${ }^{2}$ \\ ${ }^{1}$ Department of Gastroenterology and Hepatology, Mansoura Specialized Medical Hospital, Mansoura University, Mansoura, \\ ${ }^{2}$ Department of Anesthesia and Intensive Care, Mansoura University, Mansoura, Egypt
}

Background/Aims: Endoscopic ultrasound (EUS) has a limited ability to determine the nature of solid pancreatic lesions (SPLs). Most recent ultrasound processors are provided with elastography software, which allows quantification of the tissue hardness. The aim of this study is to evaluate the effectiveness of the elasticity score (ES) and strain ratio (SR) in the differentiation of benign pancreatic lesions from malignant pancreatic lesions.

Methods: The study had a retrospective design; it included 97 patients with SPLs and 19 patients with inflammatory lesions. The ES and SR were determined during the examination; finally, EUS-guided fine needle aspiration was performed.

Results: In this 2-year study, 116 patients were enrolled (97 with malignant lesions and 19 with benign lesions). There were 69 men and 47 women. Their median age was 55.9 years. A cut-off point was detected at SR of 7.75 with a specificity of $99.9 \%$, sensitivity of $90.7 \%$, positive predictive value (PPV) of $99.9 \%$, negative predictive value (NPV) of $67.9 \%$, and accuracy of $92.2 \%$. After adding the ES to the SR, the cut-off point at 7.75 resulted in a specificity of $94.6 \%$, sensitivity of $99 \%$, PPV of $98 \%$, NPV of $98.5 \%$, and accuracy of $97 \%$.

Conclusions: The use of the ES combined with the SR increases the accuracy of differentiation between benign and malignant SPLs and is an effective method for the evaluation of pancreatic masses. Clin Endosc 2019;52:360-364

Key Words: Endoscopic ultrasound; Elasticity imaging techniques; Strain ratio; Pancreas

\section{INTRODUCTION}

The diagnosis of the nature of solid pancreatic lesions (SPLs) is one of the most difficult clinical challenges. ${ }^{1}$ The limited ability of pancreatic endoscopic ultrasound (EUS) to determine the benign or malignant nature of SPLs is considered one of the major disadvantages of EUS despite the improvements in image resolution and quality. EUS-guided fine needle aspiration (EUS-FNA) is the most reliable diagnostic tool with

Received: September 7, 2018 Revised: November 1, 2018

Accepted: November 2, 2018

Correspondence: Ahmed Youssef Altonbary

Department of Gastroenterology and Hepatology, Mansoura Specialized Medical Hospital, Mansoura University, El Gomhouria St, Mit Khamis WA Kafr Al Mougi, Mansoura 35516, Egypt

Tel: +20-10-05100091, Fax: +20-50-2200878, E-mail: a.tonbary@gmail.com

ORCID: https://orcid.org/0000-0001-8850-9829

(c) This is an Open Access article distributed under the terms of the Creative Commons Attribution Non-Commercial License (http://creativecommons.org/ licenses/by-nc/3.0) which permits unrestricted non-commercial use, distribution, and reproduction in any medium, provided the original work is properly cited. a high specificity but a moderate sensitivity. EUS-FNA may yield false-negative results for malignancy and may be impossible to perform owing to technical difficulties or interposed blood vessels. As a result, efforts were made to overcome these limitations; most recent ultrasound processors are provided with a dedicated software called elastography, which allows imaging and quantification of the tissue hardness. ${ }^{2}$

The principle of elastography depends on the fact that compression of a target lesion by an echoendoscopic probe creates a strain that differs according to the hardness and softness of the tissue. Thus, it is possible to differentiate benign (soft) tissues from malignant (hard) tissues by calculating the tissue elasticity. Strain is shown via different colors based on the elasticity of the tissue: Green indicates soft tissues, whereas blue indicates hard tissues. This evaluation of tissue elasticity is qualitative. ${ }^{3}$ The quantitative analysis of tissue stiffness is now possible with the development of second-generation EUS elastography. The use of the strain ratio (SR) is the simplest method of quantifying tissue elasticity by comparing the elas- 
ticity of the target lesion with that of a selected reference area within the adjacent tissue. The most important advantage of EUS elastography is that it can provide the endoscopist with immediate data during real-time diagnostic evaluation, which can affect patients' management by assessing the nature of the lesion and more accurately targeting FNA, without the need for costly devices or software or extensive training. ${ }^{2}$

In this study, we evaluated the effectiveness of the elasticity score (ES) and SR in the differentiation of benign pancreatic lesions from malignant pancreatic lesions.

\section{PATIENTS AND METHODS}

\section{Patients}

Of 309 EUS examinations performed over the 2-year study period (from January 2016 to January 2018) at the EUS Unit of the Department of Gastroenterology of Mansoura Specialized Medical Hospital, Mansoura University (Egypt), pancreatic lesions were detected in 168 patients, 97 of them had solid-appearing pancreatic masses on EUS and were included in the study. No patient with an SPL was excluded during the study period to avoid selection bias. Nineteen patients were enrolled as controls (eight patients with chronic pancreatitis, six patients with inflammatory masses, and five patients with no pancreatic lesions who underwent EUS for extra-pancreatic causes). Twenty-two patients with cystic lesions, 18 patients with negative biopsy results, and 12 patients with missing data were excluded from the study.

The inclusion criteria were as follows: referral for EUS, definite SPLs detected on previous radiological studies, and dilated common bile duct with negative imaging study results. The exclusion criteria were as follows: cystic lesions or lesions with a predominant cystic area, contraindication to interventional endoscopy (e.g., coagulation disorders or unfitness for sedation), refusal to be involved in the study, and unknown final diagnosis. The study protocol was approved by our ethical committee, and written consents were obtained from all patients before the procedure.

\section{Methods}

The study was designed as a retrospective study to evaluate the effectiveness of the ES and SR in diagnosing SPLs. On the day of the procedure, eligible patients went to the endoscopy room for EUS examination under intravenous propofol sedation. EUS examination was performed in all patients using the Pentax linear echoendoscope EG3870UTK (PENTAX Medical, Tokyo, Japan) attached to the Hitachi Avius ultrasound system (Hitachi Medical Systems, Tokyo, Japan). All examinations were conducted by two endosonographers (AYA and $\mathrm{HH})$. Elastography was used to evaluate the hardness of the SPLs. Theoretically, the hardness of malignant lesions is greater than that of inflammatory lesions. The hardness of the lesion is assessed by the degree of tissue distortion illustrated on a color map (from red to blue representing soft to hard areas, respectively). The ES and SR were determined during the examination, and finally, EUS-FNA was performed at the end of the procedure.

\section{Elasticity score and strain ratio}

The ES was defined as follows: ES 1 was given to homogeneous green area and indicated normal tissues. ES 2 was given to heterogeneous green area predominant and indicated inflammation or fibrosis. ES 3 was given to heterogeneous blue predominant and indicated indeterminate for malignancy. ES 4 was given to homogeneous blue and indicated malignant lesions (Fig. 1).

The SR was calculated as follows: Two areas were selected; the region of interest was selected as area (A) and the normal reference tissue as area (B). Area (B) was then divided by area (A) (Fig. 1). The final SR was calculated from the mean of the repeated measures. Subsequently, the receiver-operating characteristic (ROC) curve was used to determine the best cutoff point and to calculate the diagnostic value of the SR. The best cut-off point of the SR was also combined with the ES to calculate the diagnostic value.

\section{Statistical analysis}

IBM SPSS for Windows (version 24) was used for calculating the means of the SRs, sensitivity, specificity, positive predictive value (PPV), negative predictive value (NPV), and accuracy. The best cut-off point was selected by comparing the diagnosis made on the basis of the ES and SR with the final diagnosis obtained via the cytopathological examination of the EUS-FNA samples or after surgical excision using the ROC curve; it was then used to calculate the diagnostic value. The Shapiro-Wilk test was used to check the normality of data and Student's $t$-test and Mann-Whitney test to assess parametric and non-parametric data, respectively, with $95 \%$ confidence intervals (CIs). $P$-values of $\leq 0.05$ were considered statistically significant.

\section{RESULTS}

In this 2-year study, 116 patients were enrolled (97 with malignant lesions and 19 with benign lesions). There were 69 men and 47 women. Their median age was 55.9 years (range, 12-78 years). The size, site, and final diagnosis of the pancreatic lesions, ES, and SR are shown in Tables 1 and 2. 


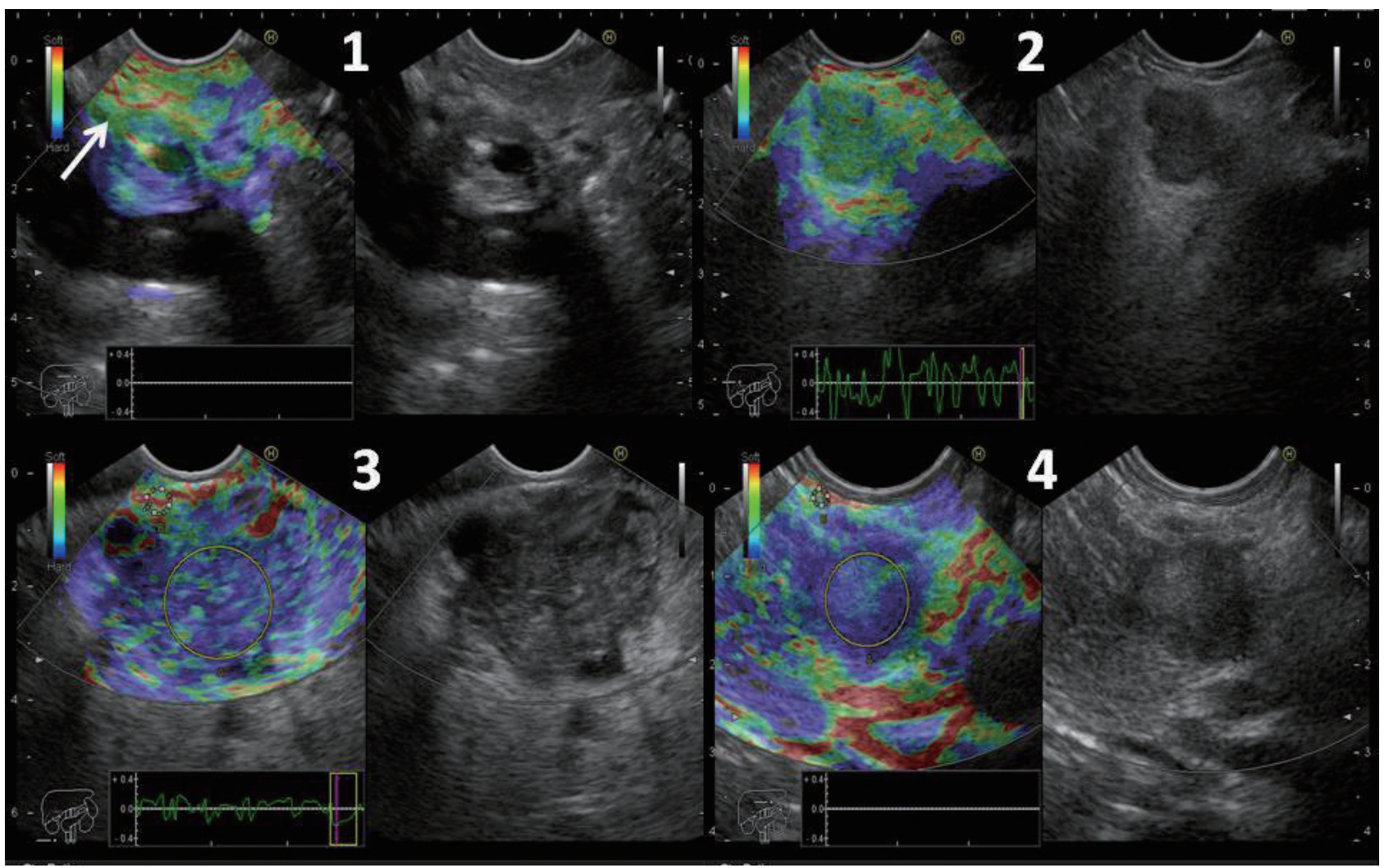

Fig. 1. Elasticity score (ES) 1 was given to homogeneous green area and indicated normal tissue (arrow). ES 2 was given to heterogeneous green area predominant and indicated fibrosis or inflammation. ES 3 was given to heterogeneous blue predominant and indicated indeterminate for malignancy. ES 4 was given to homogeneous blue and indicated malignant lesions. The strain ratio was calculated in ES 3 and 4 .

Table 1. Size, Site and Diagnosis of the Solid Pancreatic Lesions

\begin{tabular}{|c|c|c|c|}
\hline & & \multicolumn{2}{|c|}{ Number of cases $=97$} \\
\hline \multirow[t]{4}{*}{ Size } & Mean \pm SD & \multicolumn{2}{|c|}{$4.1 \pm 1.4$} \\
\hline & Median & \multicolumn{2}{|c|}{4.0} \\
\hline & Range & \multicolumn{2}{|c|}{$1.5-11.0$} \\
\hline & & Frequency & Percent \\
\hline \multirow[t]{4}{*}{ Site } & Head of the pancreas & 51 & $51.5 \%$ \\
\hline & Body of the pancreas & 29 & $29.9 \%$ \\
\hline & Uncinate process & 16 & $16.5 \%$ \\
\hline & Tail of the pancreas & 2 & $2.1 \%$ \\
\hline \multirow[t]{6}{*}{ Diagnosis } & Adenocarcinoma & 78 & $80.4 \%$ \\
\hline & Atypical & 8 & $8.2 \%$ \\
\hline & NET & 4 & $4.1 \%$ \\
\hline & SPN & 3 & $3.1 \%$ \\
\hline & Undifferentiated carcinoma & 2 & $2.1 \%$ \\
\hline & Mixed (adenocarcinoma, NET) & 2 & $2.1 \%$ \\
\hline
\end{tabular}

NET, neuroendocrine tumor; SD, standard deviation; SPN, solid pseudopapillary neoplasm.

ES 1 and 2 were deemed benign, while ES 3 and 4 were deemed malignant. The ES alone had a specificity of $89.5 \%$, sensitivity of $99 \%$, PPV of $98 \%$, NPV of $94.4 \%$, and accuracy of $97.4 \%$ (Table 3).

There was a significant difference between the mean SRs for benign SPLs (2.8) and malignant SPLs (29.4) ( $p<0.0001)$. Based 
Table 2. Strain Ratio and Elasticity Score of All Pancreatic Lesions

\begin{tabular}{|c|c|c|c|c|c|c|c|}
\hline & & \multicolumn{2}{|c|}{$\begin{array}{l}\text { All lesions } \\
(n=116)\end{array}$} & \multicolumn{2}{|c|}{$\begin{array}{c}\text { Benign lesions } \\
\quad(n=19)\end{array}$} & $\begin{array}{c}\text { Malignant lesions } \\
(n=97)\end{array}$ & $p$-value \\
\hline \multirow[t]{5}{*}{ Strain ratio } & Mean \pm SD & \multicolumn{2}{|c|}{$25.0 \pm 28.4$} & \multicolumn{2}{|c|}{$2.8 \pm 1.2$} & $29.4 \pm 29.1$ & $<0.0001$ \\
\hline & Median & \multicolumn{2}{|c|}{16.2} & \multicolumn{2}{|c|}{2.8} & 19.7 & - \\
\hline & Range & \multicolumn{2}{|c|}{ 1.2: 164} & \multicolumn{2}{|c|}{$1.2: 5.6$} & 3.6: 164 & - \\
\hline & & \multicolumn{2}{|c|}{$\begin{array}{l}\text { All lesions } \\
(n=116)\end{array}$} & \multicolumn{2}{|c|}{$\begin{array}{l}\text { Benign lesions } \\
\quad(n=19)\end{array}$} & \multicolumn{2}{|c|}{$\begin{array}{l}\text { Malignant lesions } \\
\qquad(n=97)\end{array}$} \\
\hline & & Frequency & Percent & Frequency & Percent & Frequency & Percent \\
\hline \multirow[t]{4}{*}{ Elasticity score } & ES 1 & 2 & $1.7 \%$ & - & - & 2 & $10.5 \%$ \\
\hline & ES 2 & 16 & $13.8 \%$ & 1 & $1 \%$ & 15 & $78.9 \%$ \\
\hline & ES 3 & 95 & $81.9 \%$ & 93 & $95.9 \%$ & 2 & $10.5 \%$ \\
\hline & ES 4 & 3 & $2.6 \%$ & 3 & $3.1 \%$ & - & - \\
\hline
\end{tabular}

ES, elasticity score; SD, standard deviation.

Table 3. Diagnostic Values of Elasticity Score and Strain Ratio

\begin{tabular}{lcccc}
\hline & ES & SR 4.55 & SR 7.75 & ES \& SR 7.75 \\
\hline Sensitivity & $99 \%$ & $96.9 \%$ & $90.7 \%$ & $99 \%$ \\
Specificity & $89.5 \%$ & $94.7 \%$ & $99.9 \%$ & $94.6 \%$ \\
PPV & $98 \%$ & $98.9 \%$ & $99.9 \%$ & $98 \%$ \\
NPV & $94.4 \%$ & $85.7 \%$ & $67.9 \%$ & $98.5 \%$ \\
Accuracy & $97.4 \%$ & $96.5 \%$ & $92.2 \%$ & $97 \%$ \\
\hline
\end{tabular}

ES, elasticity score; NPV, negative predictive value; PPV, positive predictive value; SR, strain ratio.

on the ROC curve analysis results, the best cut-off point of the SR to obtain the maximum area under the curve was 4.55 with a specificity of $94.7 \%$, sensitivity of $96.9 \%$, PPV of $98.9 \%$, NPV of $85.7 \%$, and accuracy of $96.5 \%$. Another cut-off point was detected at 7.75 with a specificity of $99.9 \%$, sensitivity of $90.7 \%$, PPV of $99.9 \%$, NPV of $67.9 \%$, and accuracy of $92.2 \%$. After adding the ES to the SR, the cut-off point at 7.75 resulted in a specificity of $94.6 \%$, sensitivity of $99 \%$, PPV of $98 \%$, NPV of $98.5 \%$, and accuracy of $97 \%$ (Table 3).

\section{DISCUSSION}

The current study provides evidence supporting EUS elastography as a useful and accurate tool for differentiating the nature of solid pancreatic masses. The SR adds important and objective data to EUS, supporting the benign or malignant nature of the mass, by providing a quantitative analysis of tissue stiffness. ${ }^{4,5}$ The SR provides a cut-off point for the differentiation between benign and malignant masses. Based on these results, and by using a well-defined reference method, EUS elastography can be used to define the benign or malignant nature of solid pancreatic masses objectively with high accuracy.

The high rate of false-negative results of EUS-FNA (up to 15\%-17\%) makes its diagnostic accuracy questionable. ${ }^{6}$ Further, EUS-FNA has many disadvantages, including iatrogenic complications, need for repeated needle passes to acquire an adequate tissue sample, ${ }^{7}$ high learning curve, and high level of experience to obtain satisfactory results.

These disadvantages raised the necessity to improve other techniques for the diagnosis of solid pancreatic masses with fewer complications and higher efficacy. Dawwas et al. reported a sensitivity of $100 \%$ for the ES; however, it had a very low specificity of $16.7 \%{ }^{8}$ This was in contrast to the results of other published studies ${ }^{4,9}$ and our study that showed a specificity of $89.5 \%$. In our study, two patients with ES 3 were finally diagnosed with inflammatory masses; this high ES may be attributed to the presence of fibrous tissues or calcifications. However, the subjectivity of the ES appeared to be a problem when using this technique.

The SR with different cut-off points was evaluated in many studies to overcome the subjectivity and increase the specificity of EUS elastography. ${ }^{4,10}$ We found a cut-off point of 4.55 , which had specificity, sensitivity, PPV, NPV, and accuracy of $94.7 \%, 96.9 \%, 98.9 \%, 85.7 \%$, and $96.5 \%$, respectively. This was 
similar to the results of the study by Okasha and colleagues, ${ }^{11}$ who reported a cut-off point of 3.8, which had NPV, sensitivity, and accuracy of $86 \%, 99 \%$, and $96 \%$, respectively, but lower specificity and PPV of 53\% and $84 \%$, respectively. Kongkam et al. ${ }^{12}$ also reported a cut-off point of 3.17, which had lower specificity, sensitivity, PPV, NPV, and accuracy of $66.7 \%$, $86.2 \%, 89.3 \%, 60 \%$, and $81.6 \%$, respectively. In our study, the best cut-off point for the differentiation between malignant and benign SPLs was 7.75. The SR of 7.75 had a specificity of $99.9 \%$, sensitivity of $90.7 \%$, PPV of $99.9 \%$, NPV of $67.9 \%$, and accuracy of $92.2 \%$. The low SR in some malignant lesions may be attributed to degeneration or necrosis within the lesion.

In our study, the SRs were calculated for 97 consecutive patients with SPLs (78 with adenocarcinomas, eight with atypical lesions, four with neuroendocrine tumors, three with solid pseudopapillary tumors, two with undifferentiated carcinomas, and two with mixed adenocarcinomas with neuroendocrine tissue) and 19 patients with benign lesions. The SR was significantly higher among the patients with malignant pancreatic masses than among those with inflammatory masses. The inflammatory lesions exhibited an SR (mean, 2.8; 95\% CI, $1.2-5.6)$ that was significantly lower than that of the pancreatic malignant lesions (mean, 29.4; 95\% CI, 3.6-164; $p<0.0001$ ). These results were close to those of the study by Iglesias-Garcia et al., ${ }^{13}$ who investigated the SR of 86 patients with SPLs (49 with adenocarcinomas, 27 with inflammatory lesions, six with neuroendocrine tumors, two with metastatic lesions from lung cancers, one with solid pseudopapillary neoplasm, and one with lymphoma) and 20 controls. The malignant pancreatic masses showed a significantly higher SR than did the inflammatory masses. The SR of the inflammatory masses (3.28) was significantly lower than that of the pancreatic adenocarcinomas (18.12); however, it was higher than that of the normal pancreatic tissues $(p<0.001)$.

To increase the efficacy of differentiating malignant SPLs from benign SPLs, we combined the ES with the SR cut-off point of 7.75 to achieve a specificity of $94.6 \%$, sensitivity of $99 \%$, PPV of $98 \%$, NPV of $98.5 \%$, and accuracy of $97 \%$, which is better than the use of each technique alone.

Elastography may have some limitations including as follows: Compression of the tissue by the echoendoscope is difficult to control; the motion artifacts created by respiratory and cardiac movements and some structures with very low or very high stiffness, such as major vessels or spine, are difficult to exclude from the region of interest. Most of these limitations can be reduced by experienced endoscopists. In our study, a stable elastography image for 5 seconds was required for elasticity measurement to minimize these limitations.

Despite all these theoretical limitations, we found that the use of the ES combined with the SR increases the accuracy of differentiation between benign and malignant SPLs and is an effective method for the evaluation of pancreatic masses.

\section{Conflicts of Interest}

The authors have no financial conflicts of interest.

\section{REFERENCES}

1. Chantarojanasiri T, Kongkam P. Endoscopic ultrasound elastography for solid pancreatic lesions. World J Gastrointest Endosc 2017;9:506-513.

2. Deprez PH. EUS elastography: is it replacing or supplementing tissue acquisition? Gastrointest Endosc 2013;77:590-592.

3. Arcidiacono PG. Endoscopic ultrasound elastography. Gastroenterol Hepatol (N Y) 2012;8:48-67.

4. Iglesias-Garcia J, Larino-Noia J, Abdulkader I, Forteza J, Dominguez-Munoz JE. Quantitative endoscopic ultrasound elastography: an accurate method for the differentiation of solid pancreatic masses. Gastroenterology 2010;139:1172-1180.

5. Lee TH, Cho YD, Cha SW, et al. Endoscopic ultrasound elastography for the pancreas in Korea: a preliminary single center study. Clin Endosc 2013;46:172-177.

6. Yamabe A, Irisawa A, Bhutani MS, et al. Efforts to improve the diagnostic accuracy of endoscopic ultrasound-guided fine-needle aspiration for pancreatic tumors. Endosc Ultrasound 2016;5:225-232.

7. Eloubeidi MA, Tamhane A, Varadarajulu S, Wilcox CM. Frequency of major complications after EUS-guided FNA of solid pancreatic masses: a prospective evaluation. Gastrointest Endosc 2006;63:622-629.

8. Dawwas MF, Taha H, Leeds JS, Nayar MK, Oppong KW. Diagnostic accuracy of quantitative EUS elastography for discriminating malignant from benign solid pancreatic masses: a prospective, single-center study. Gastrointest Endosc 2012;76:953-961.

9. Giovannini M. Endoscopic ultrasound elastography. Pancreatology 2011;11 Suppl 2:34-39.

10. Giovannini M, Thomas B, Erwan B, et al. Endoscopic ultrasound elastography for evaluation of lymph nodes and pancreatic masses: a multicenter study. World J Gastroenterol 2009;15:1587-1593.

11. Okasha H, Elkholy S, El-Sayed R, et al. Real time endoscopic ultrasound elastography and strain ratio in the diagnosis of solid pancreatic lesions. World J Gastroenterol 2017;23:5962-5968.

12. Kongkam P, Lakananurak N, Navicharern P, et al. Combination of EUSFNA and elastography (strain ratio) to exclude malignant solid pancreatic lesions: a prospective single-blinded study. J Gastroenterol Hepatol 2015;30:1683-1689.

13. Iglesias-Garcia J, Lindkvist B, Lariño-Noia J, Domínguez-Muñoz JE. Endoscopic ultrasound elastography. Endosc Ultrasound 2012;1:8-16. 